Manuelle Medizin 2007 • 45:17-20 DOI 10.1007/s00337-006-0485-6

Online publiziert: 24. November 2006

(c) Springer Medizin Verlag 2006

Y. Robinson - C. E. Heyde · R. Kayser

Klinik für Unfall- und Wiederherstellungschirurgie, Charité -

Universitätsmedizin Berlin Campus Benjamin Franklin, Berlin

\title{
Diagnostik und Therapie der thorakalen Spondylodiszitis
}

gischen Infektsanierung ist, wird die Stabilisierung, insbesondere die Verwendung von ventralen Implantaten, kontrovers diskutiert [13].

\section{Pathogenese}

Üblicherweise erfolgt die hämatogene Infektion der Bandscheibe aus den endplattennahen Gefäßplexus durch bakteriell besiedelte Mikroembolie aus wirbelsäulenfernen Infektherden [23]. Über die ventrale Gefäßanastomose kann sich eine Infektion aus der kaudalen zur kranialen Endplatte [16] und von dort in die beim Erwachsenen kaum vaskularisierte und daher kaum immunkompetente Bandscheibe ausbreiten [6]. Bei Kindern ist jedoch eine direkte hämatogene Besiedlung der hochvaskularisierten Bandscheibe möglich [2]. Auch bei hochgradig degenerierten Bandscheiben ist eine sekundäre Gefäßeinsprossung beschrieben, die eine direkte hämatogene Besiedlung ermöglicht [19]. Prädisponiert für Spondylodiszitiden sind immunsupprimierte Patienten (rheumatoide Arthritis, AIDS etc.), Diabetiker und Patienten mit Hämodialyse [13].

Aufgrund der stetig steigenden Zahl an spinalen Infiltrationen und Wirbelsäuleneingriffen werden wir immer häufiger mit postoperativen Infektionen der Wirbelsäule konfrontiert. Zwischen 0,2 und $4 \%$ aller Wirbelsäuleneingriffe führen $\mathrm{zu}$ Infektionen. Diese machen immerhin bis zu 30\% aller Spondylodiszitiden aus [21]. Darüber hinaus sind Fälle mit thorakaler Spondylodiszitis nach penetrierendem Thoraxtrauma beschrieben [10].

\section{Epidemiologie}

Die lumbale (LWK 2-SWK 1) und thorakolumbale (BWK 10-LWK 2) Wirbelsäule sind Hauptmanifestationsorte der Spondylodiszitis. Zervikale (HWK 1BWK 1) Spondylodiszitiden sind dagegen sehr selten. Thorakale Spondylodiszitiden (BWK 1-10) entdeckten Beronius et al. [1] in $27 \%$ ihrer Patienten ( $n=58)$. Lilienqvist et al. [15] fanden in $20 \%(\mathrm{n}=20)$ und die Arbeitsgruppe Lerner in $29 \%$ [13] und $30 \%$ [14] ihrer untersuchten Fälle thorakale Spondylodiszitiden $(n=62)$. Isenberg et al. [11] beschrieben sie bei $27 \%$ der Patienten $(n=34)$.

Der häufigste Erreger der Spondylodiszitis ist in $55-80 \%$ der Fälle Staphylococcus aureus [19], gefolgt von Staphylococcus epidermidis, Escherichia coli, Pseudomonaden und Streptokokken. In Europa macht die tuberkulöse Infektion der thorakalen Wirbelsäule einen kleinen Teil der Infektionen aus. In Entwicklungsländern macht die Wirbelsäuleninfektion ein Drittel der extrapulmonalen Manifestationen der Tuberkulose aus [24].

Auch Candida albicans und Aspergillus sind als Erreger von Spondylodiszitiden beschrieben [11]. Einige Autoren unterscheiden septische von aseptischen Spondylodiszitiden [7]. Letztere ist eine Ausschlussdiagnose nach blanden Punktionen oder keimfreien intraoperativen Abstrichen. Es gibt jedoch Zweifel an der Existenz einer aseptischen Spondylodiszitis und manche vermuten einen „Lowgrade-Infekt" hinter diesem Erkrankungsbild. einander. Während ein radikales Débridement fester Bestandteil jeder chirur- 


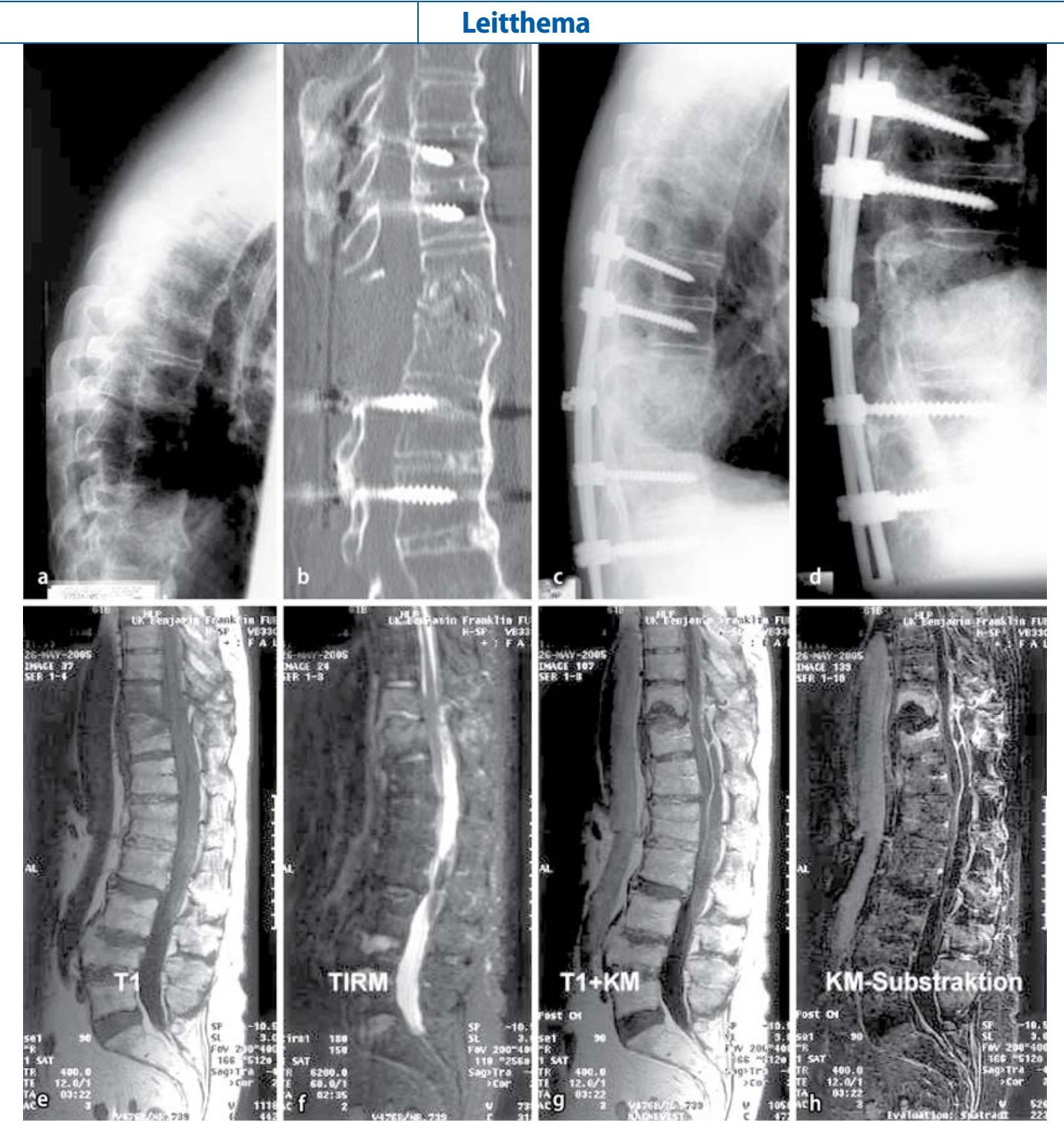

Abb. 1a-h < Ein 67-jähriger Patient wurde uns mit akuten BWS-Schmerzen bei Z.n. lleus und Pneumonie zugewiesen. Klinisch zeigte der Patient ein partielles Querschnittssyndrom sub Th12. Im Röntgenbild (a) fiel eine Erosion der Endplatten BWK10/11 auf. Im MRT (e-h) zeigte sich eine Spondylodiszitis BWK10/11 mit epiduraler Abszeßbildung. Es erfolgte daraufhin die sofortige dorsale Laminektomie BWK9-11, dorsale Instrumentation BWK8LWK2 und ventrale Beckenkammspaninterposition BWK10/11 (b-d). Unter Antibiose und neurologischer Rehabilitation kam es zu einer Rückbildung der Querschnittssymptomatik innerhalb von 3 Tagen. Zwei Jahre postoperativ ist der Patient klinisch beschwerdefrei ohne Rezidiv

\section{Diagnostik}

Patienten mit thorakaler Spondylodiszitis zeigen ein unspezifisches Krankheitsbild, das oft von multiplen Begleiterkrankungen überdeckt wird. Auffällig wird der Patient meist durch Rückenschmerzen, die in ihrer Intensität zunehmen, bis aus initial belastungsabhängigen Beschwerden Ruheschmerzen geworden sind. Darüber hinaus klagt der Patient über ein generalisiertes Krankheitsgefühl mit Schlaffheit, Gewichtsverlust, Schüttelfrost und Nachtschweiß, in einem Drittel der Fälle auch in Verbindung mit Fieber [19].

Klinisch zeigt der Patient einen Klopfschmerz über der betroffenen Region mit schmerzhaft eingeschränktem Bewegungsumfang des entsprechenden Segments. Neurologisch sind die meisten $\mathrm{Pa}$ tienten unauffällig. Treten jedoch intraspi- nale Abszedierungen der BWS auf, kann es zu neurologischen Komplikationen mit Querschnittsymptomatik kommen.

Laborchemisch zeigt sich lediglich in $43 \%$ der Patienten mit Spondylodiszitis eine erhöhte Leukozytenzahl [9]. Das C-reaktive Protein (CRP) zeigt dagegen eine Sensitivität von $64-100 \%$ und eine Spezifität von $62-95,8 \%[5,18]$. Postoperativ muss natürlich der Verlauf des CRP, der seinen Gipfel am 2.-3. Tag hat und innerhalb von 14 Tagen auf den Normalwert zurückkehrt, berücksichtigt werden [5]. Die weit verbreitete Messung der Blutsenkungsgeschwindigkeit (BSG) hat eine Sensitivität von 78,1$82 \%$ und eine Spezifität von 38,1-62\% [5, 18]. Auch hier ist postoperativ ein Gipfel am 4. Tag zu beachten [12].

Das konventionelle Röntgenbild (• Abb. 1a) hinkt den klinischen Symptomen meist hinterher. Erste konventionell-radi- ologische Zeichen werden frühestens 34 Wochen nach Infektionsbeginn durch Aufweichung der Konturen der Endplatten gesehen [19]. Nach 4-8 Wochen lassen sich zunehmend reaktive knöcherne Anbauten an den Endplatten nachweisen. Dann tritt mehr und mehr eine Lyse der Endplatten mit konsekutiver kyphotischer Deformität in den Vordergrund. Die Computertomografie kann zusätzliche Informationen zu knöchernen Destruktionen der Endplatten liefern. Außerdem lassen sich CT-gesteuerte Punktionen zur mikrobiologischen Diagnostik durchführen. Zur Beurteilung der intra- und paraspinalen Weichteile sowie des Knochenödems ist die Magnetresonanztomografie (MRT) das Mittel der Wahl (• Abb. 1e-h). Mit einer Sensitivität von 93\% und einer Spezifität von $97 \%$ zeichnet sie sich vor allen anderen Verfahren aus [22]. Heutzutage wer- 
den kontrastmittelunterstützte Verfahren verwendet, um das Ausmaß der Infektion darstellen zu können [16]. Sollte ein MRTGerät nicht verfügbar sein oder Stahlimplantate bzw. Herzschrittmacher eine solche Untersuchung verbieten, kann eine Skelettszintigraphie durchgeführt werden. Gallium-(Ga-)67-Untersuchungen weisen eine Sensitivität von $89 \%$ und eine Spezifität von $85 \%$ auf [3]. Durch die Kombination von Ga-67-Szintigraphie mit Einzelphotonenemissionscomputertomografie (SPECT) lässt sich eine Sensitivität von $92 \%$ und eine Spezifität von $92 \%$ erreichen [17]. Szintigraphisch kann jedoch nicht zwischen einem knöchernen oder einem Weichteilprozess unterschieden werden. Alternativ wird vermehrt die Verwendung der Positronenemissionstomografie (PET) diskutiert. Diese soll eine mit der MRT vergleichbare Sensitivität und Spezifität haben [25].

Differenzialdiagnostisch müssen sämtliche Formen der erosiven Osteochondrose oder der destruktiven Spondylarthopathie berücksichtigt werden [24].

Durchgesetzt hat sich der Algorithmus der North American Spine Society [21]:

1. Die laborchemische Diagnostik schließt Leukozytenzahl, Differenzialblutbild und BSG oder CRP ein.

2. Bei erhöhten Infektparametern sollten Blutkulturen abgenommen werden.

3. Bei negativen Blutkulturen sollte eine CT-gesteuerte Probeentnahme erwogen werden.

4. Bei Keimnachweis sollte eine resistenzgerechte, bei fehlendem Keimnachweis eine Breitspektrumantibiose für 12 Wochen erfolgen, zusätzlich Immobilisation im starren Mieder.

5. Sollte nach 6 Wochen Antibiose eine Infektpersistenz bestehen, sollte ein chirurgisches Débridement erwogen werden.

6. Bei paraspinalen Abszedierungen besteht eine dringliche Operationsindikation.

\section{Therapie}

Ziele der operativen Infektsanierung sind die komplette Entfernung der infizierten Bandscheibe, das Débridement des infizierten und nekrotischen Gewebes und die Stabilisierung mit einem Knochentransplantat. Bei größeren Defekten mit kyphotischer Fehlstellung sind eine Deformitätenkorrektur und eine Instrumentation notwendig.

Der Zugang zur thorakalen Bandscheibe ist üblicherweise transthorakal über eine laterale Thorakotomie. Alternativ kann auch ein thorakoskopischer Eingriff erwogen werden, jedoch ist unserer Erfahrung nach für ein radikales Débridement und eine ventrale Fusion ein größerer $\mathrm{Zu}$ gang notwendig. Nach Resektion der Bandscheibe sollte der Spinalkanal von ventral dekomprimiert werden, um mögliche neurologische Komplikationen $\mathrm{zu}$ vermeiden oder einen epiduralen Abszess zu drainieren. Nach Débridement der Endplatten des Bandscheibenfaches können als Interponat Rippenspäne, Beckenkammspäne oder mit Beckenkammspongiosa gefüllte Titankörbe zur ventralen Stabilisierung verwendet werden. Zum Einsatz metallischer Implantate bei Spondylodiszitiden gehen die Meinungen jedoch weit auseinander. Die ventrale Säule wird durch die Interposition eines Titankorbs stabilisiert. Die Ruhigstellung ermöglicht eine optimierte Ausheilung (• Abb. 1b-d). Der bakterielle Biofilm scheint jedoch auch an Titanimplantaten zu adhärieren [8]. Dennoch wurde bereits mehrfach über eine erfolgreiche Verwendung von Titanimplantaten in der ventralen Säule berichtet [4, 11, 15].

Bei intraspinalen Abszessen und bei mehrsegmentalem Befall ist ein zusätzlicher dorsaler Eingriff indiziert. Dieser kann einzeitig mit dem ventralen Eingriff oder zweizeitig erfolgen. Bei relevanten Stenosen lässt sich hier eine dorsale Dekompression und außerdem eine Abszessdrainage durchführen. Deformitäten und Instabilität können durch dorsale Instrumentationen behoben werden, um eine frühzeitige Belastbarkeit der infizierten Wirbelsäule zu erreichen. Auch hier können minimalinvasive Techniken eingesetzt werden, jedoch sind diese mit höheren vaskulären Komplikationen vergesellschaftet und die Drainage eines epiduralen Abszesses ist erschwert [9]. Bei erfolgter dorsaler Instrumentierung ist eine orthetische Versorgung nicht notwendig, ansonsten ist sie fester Bestandteil der Nachbehandlung.
Manuelle Medizin 2007 · 45:17-20

DOI 10.1007/s00337-006-0485-6

○) Springer Medizin Verlag 2006

Y. Robinson · C. E. Heyde - R. Kayser Diagnostik und Therapie der thorakalen Spondylodiszitis

\section{Zusammenfassung}

Aufgrund der zunehmenden Anzahl multimorbider Patienten sowie der Zuwanderung aus Entwicklungsländern ist kein Rückgang der Inzidenz der thorakalen Spondylodiszitis festzustellen. Die Diagnostik umfasst die Klinik des Patienten, das Labor und die radiologische Diagnostik mit Röntgen und Magnetresonanztomografie. Zur chirurgischen Sanierung gehört neben dem radikalen Débridement auch die Deformitätenkorrektur und adäquate Stabilisierung, wobei die Verwendung von Titanimplantaten kontrovers diskutiert wird. Adjuvant muss eine resistenzgerechte Antibiose durchgeführt werden.

\section{Schlüsselwörter}

Chirurgische Sanierung · Radikales Débridement - Deformitätenkorrektur - Titanimplantate $\cdot$ Resistenzgerechte Antibiose

\section{Diagnostic work-up and treatment of thoracic spondylodiscitis}

\section{Abstract}

Due to the increase in patients with multiple co-morbidities and immigration from developing countries the incidence of thoracic spondylodiscitis has not decreased. The diagnosis of thoracic spondylodiscitis is based on clinical findings, laboratory diagnostics and radiological imaging, including $\mathrm{X}$-ray and MRI. Surgical therapy includes radical debridement, correction of deformity and adequate stabilisation, even though the use of titanium implants is discussed controversially. Additionally antibiotic therapy according to the resistance spectrum is necessary. Early diagnosis and therapy enable healing without severe complications.

\section{Keywords}

Surgical therapy · Radical debridement · Correction of deformity - Titanium implants - Antibiotic therapy 
Postoperativ ist eine resistenzgerechte adjuvante Antibiose von 12 Wochen indiziert. Einige Autoren halten eine Antibiose von 6 Wochen für ausreichend [20].

\section{Fazit für die Praxis}

Patienten mit thorakaler Spondylodiszitis zeigen ein unspezifisches Krankheitsbild, das oft von multiplen Begleiterkrankungen überdeckt wird. Da die septische Spondylodiszitis untherapiert eine fortschreitende destruierende Erkrankung ist, sollte die Diagnose möglichst früh gestellt und eine Therapie sofort eingeleitet werden. Dabei hat sich der Algorithmus der North American Spine Society durchgesetzt. Ziele der operativen Infektsanierung sind die komplette Entfernung der infizierten Bandscheibe, das Débridement des infizierten und nekrotischen Gewebes sowie die Stabilisierung mit einem Knochentransplantat. Die konsequente resistenzgerechte adjuvante Langzeitantibiose ermöglicht ein rezidivfreies Ausheilen der Infektion.

\section{Korrespondierender Autor \\ Dr. Y. Robinson}

Klinik für Unfall- und Wiederherstellungschirurgie, Charité - Universitätsmedizin Berlin Campus Benjamin Franklin Hindenburgdamm 30, 12200 Berlin yohan.robinson@charite.de

Interessenkonflikt. Es besteht kein Interessenkonflikt. Der korrespondierende Autor versichert, dass keine Verbindungen mit einer Firma, deren Produkt in dem Artikel genannt ist, oder einer Firma, die ein Konkurrenzprodukt vertreibt, bestehen. Die Präsentation des Themas ist unabhängig und die Darstellung der Inhalte produktneutral.

\section{Literatur}

1. Beronius M, Bergman B, Andersson R (2001) Vertebral osteomyelitis in Goteborg, Sweden: a retrospective study of patients during 1990-95. Scand J Infect Dis 33: 527-532

2. Brown R, Hussain M, McHugh K, Novelli V, Jones D (2001) Discitis in young children. J Bone Joint Surg $\mathrm{Br} 83: 106-111$

3. Bruschwein DA, Brown ML, McLeod RA (1980) Gallium scintigraphy in the evaluation of disk-space infections: concise communication. J Nucl Med 21: 925-927

4. Eysel P, Hopf C, Vogel I, Rompe JD (1997) Primary stable anterior instrumentation or dorsoventral spondylodesis in spondylodiscitis? Results of a comparative study. Eur Spine J 6: 152-157
5. Fouquet B, Goupille P, Jattiot F et al. (1992) Discitis after lumbar disc surgery. Features of "aseptic" and "septic" forms. Spine 17: 356-358

6. Gibson MJ, Karpinski MRK, Slack RCB, Cowlishaw WA, Webb JK (1987) The penetration of antibiotics into the normal intervertebral disc. J Bone Joint Surg 69B: 784-786

7. Grane P, Josephsson A, Seferlis A, Tullberg T (1998) Septic and aseptic post-operative discitis in the lumbar spine - evaluation by MR imaging. Acta Radiol 39: 108-115

8. Ha KY, Chung YG, Ryoo SJ (2005) Adherence and biofilm formation of staphylococcus epidermidis and mycobacterium tuberculosis on various spinal implants. Spine 30: 38-43

9. Hadjipavlou AG, Mader JT, Necessary JT, Muffoletto $A J(2000)$ Hematogenous pyogenic spinal infections and their surgical management. Spine 25: 1668-1679

10. Heary RF, Vaccaro AR, Mesa JJ, Balderston RA (1996) Thoracolumbar infections in penetrating injuries to the spine. Orthop Clin North Am 27: 69-81

11. Isenberg J, Jubel A, Hahn U, Seifert H, Prokop A (2005) Die mehrzeitige Spondylodese. Orthopäde 34: $159-66$

12. Jonsson B, Soderholm R, Stromqvist B (1991) Erythrocyte sedimentation rate after lumbar spine surgery. Spine 16: 1049-1050

13. Lerner T, Hackenberg L, Rösler S et al. (2005) Operative Therapie der unspezifischen und spezifischen Spondylodiszitis. Z Orthop 143: 204-212

14. Lerner T, Schulter T, Bullmann V et al. (2006) Anterior column reconstruction using titanium ring cages in severe vertebral osteomyelitis. Eur J Trauma 32: 227-237

15. Liljenqvist U, Lerner T, Bullmann V et al. (2003) Titanium cages in the surgical treatment of severe vertebral osteomyelitis. Eur Spine J 12: 606-612

16. Longo M, Granata F, Ricciardi K, Gaeta M, Blandino A (2003) Contrast-enhanced MR imaging with fat suppression in adult-onset septic spondylodiscitis. Eur Radiol 13: 626-637

17. Love C, Patel M, Lonner BS, Tomas MB, Palestro CJ (2000) Diagnosing spinal osteomyelitis: a comparison of bone and Ga-67 scintigraphy and magnetic resonance imaging. Clin Nucl Med 25: 963-977

18. Meyer B, Schaller K, Rohde V, Hassler W (1995) The C-reactive protein for detection of early infections after lumbar microdiscectomy. Acta Neurochir (Wien) 136: 145-150

19. Müller EJ, Russe OJ, Muhr G (2004) Osteomyelitis der Wirbelsäule. Orthopäde 33: 305-315

20. Przybylski GJ, Sharan AD (1991) Single-stage autogenous bone grafting and internal fixation in the surgical management of pyogenic discitis and vertebral osteomyelitis. J Neurosurg Spine 94: 1-7

21. Silber JS, Anderson DG, Vaccaro AR, Anderson PA McCormick P (2002) Management of postprocedural discitis. Spine J 2: 279-287

22. Szypryt EP, Hardy JG, Hinton CE, Worthington BS, Mulholland RC (1988) A comparison between magnetic resonance imaging and scintigraphic bone imaging in the diagnosis of disc space infection in an animal model. Spine 13: 1042-1048

23. Tay BKB, Deckey J, Hu SS (2002) Spinal infections. J Am Acad Orthop Surg 10: 188-197

24. Weyreuther M, Heyde CE, Westphal M, Zierski I, Weber U (2006) MRT-Atlas Orthopädie und Neurochirurgie - Wirbelsäule. Springer, Berlin Heidelberg New York

25. Zhuang H, Duarte PS, Pourdehand M, Shnier D, Alavi A (2000) Exclusion of chronic osteomyelitis with F-18 fluorodeoxyglucose positron emission tomographic imaging. Clin Nucl Med 25: 281-284

\section{Training und Psychotherapie helfen auch älteren Schmerz- patienten}

Chronische Rückenschmerzen können auch bei älteren Menschen mit einem Trainingsprogramm erfolgreich behandelt werden, das nicht nur auf körperliche, sondern auch auf psychische Ursachen abzielt. Eine Untersuchung der Orthopädischen Universitätsklinik Heidelberg hat jetzt gezeigt, dass rund zwei Drittel der Teilnehmer an einem solchen integrativen Programm in der Altersgruppe zwischen 50 und 65 Jahren ihre Arbeit wieder aufnehmen konnten - in vergleichbarem Umfang wie jüngere Schmerzpatienten.

Frühere Studien des Instituts haben gezeigt, dass die Behandlung von psychischer Belastung und Stress Patienten beweglicher und arbeitsfähiger macht. Die aktuelle Untersuchung belegt erstmals, dass dies nicht nur für jüngere Menschen gilt.

An der Studie nahmen insgesamt 405 Männer und Frauen teil, die seit mindestens drei Monaten an Rückenschmerzen litten und bereits sechs Wochen krank geschrieben waren. Alle Patienten nahmen an einem dreiwöchigen multidisziplinären GanztagesTherapieprogramm teil. Das Programm umschloss krankengymnastische Übungen und Rückentraining ebenso wie Entspannungsübungen, Psychotherapie und Anpassungen am Arbeitsplatz. Wesentliche Beobachtung bei der Studie war: Schmerzrückbildung und Steigerung der Lebensqualität konnte bei den älteren Patienten erreicht werden, ohne dass die Rückenfunktionen verbessert wurden.

\section{Quelle:}

Orthopädische Universitätsklinik Heidelberg, www.uni-heidelberg.de

\section{Originalpublikation:}

Buchner M, Neubauer E, Zahlten-Hinguranage A, Schiltenwolf M (2006) Age as a predicting factor in the therapy outcome of multidisciplinary treatment of patients with chronic low back pain-a prospective longitudinal clinical study in 405 patients. Clin Rheumatol. Jul 25 